\title{
Orexin 2 receptor as a potential target for immunotoxin and antibody-drug conjugate cancer therapy
}

\author{
MASATO KISHIDA $^{1}$, KAZUNORI ISHIGE ${ }^{2}$, TOMOHISA HORIBE ${ }^{3}$, NORIKO TADA ${ }^{3}$, \\ NOBUTAKA KOIBUCHI ${ }^{4}$, JUNICHI SHODA ${ }^{2}$, KIYOSHI KITA ${ }^{1}$ and KOJI KAWAKAMI ${ }^{3}$
}

\author{
${ }^{1}$ Department of Biomedical Chemistry, Graduate school of Medicine, The University of Tokyo, Tokyo 113-0033; \\ ${ }^{2}$ Department of Gastroenterology and Hepatology, Institute of Clinical Medicine, University of Tsukuba, Tsukuba 305-8575; \\ ${ }^{3}$ Department of Pharmacoepidemiology, Graduate School of Medicine and Public Health, Kyoto University, Kyoto 606-8501; \\ ${ }^{4}$ Department of Pharmacology and Molecular Therapeutics, Kumamoto University \\ Graduate School of Medical Sciences, Kamamoto 860-8556, Japan
}

Received August 1,2011; Accepted November 15, 2011

DOI: $10.3892 / \mathrm{ol} .2011 .528$

\begin{abstract}
Targeting tumor-specific receptors is a promising approach for cytotoxic agents. The orexin 2 receptor (OX2R) has reportedly been expressed in a few types of cancer, but not in normal, cells. This study aimed to explore and assess the expression levels of OX2R in a wide range of cancer cell lines and clinical samples to identify its localization. To analyze OX2R expression, we developed a polyclonal antibody specific to OX2R by immunizing two rabbits with a peptide cocktail. A total of 36 cancer cell lines were employed for reverse transcription polymerase chain reaction (RT-PCR) and western blot analysis, and 221 samples from various tissue arrays were used for the immunohistochemistry of OX2R expression. OX2R was identified in three cancerous cell lines, from the gallbladder, squamous cell carcinoma of the head and neck (SCCHN) and glioblastoma. With clinical samples of tissue arrays, 69/221 (31.2\%) samples reacted positively with the OX2R antibody. We confirmed its presence on the cell membrane. In conclusion, OX2R was identified on several cancer cells as well as clinical samples. Further studies with larger numbers of clinical samples are required to confirm the statistical significance of the presence and relationships of OX2R with tumor histology. Results of the current study suggested that OX2R is a potent target for immunotoxin or antibody-drug conjugate (ADC) cancer therapy on OX2Rpositive cancer cells.
\end{abstract}

Correspondence to: Dr Koji Kawakami, Department of Pharmacoepidemiology, Graduate School of Medicine and Public Health, Kyoto University, Yoshidakonoecho, Sakyoku, Kyoto 606-8501, Japan

E-mail: kawakami.koji.4e@kyoto-u.ac.jp

Key words: orexin 2 receptor, immunotoxin, antibody-drug conjugates, molecular target therapy

\section{Introduction}

Cytotoxic drugs are still widely used to treat malignant tumors via tumor cell death by apoptosis (1). One of the most representative cytotoxins is cisplatin, which reacts with target genomic DNA to form DNA adducts (2). Cytotoxins are typically non-selective and this lack of selectivity occasionally results in significant toxicity to normal cells. Moreover, treatment of cancer patients with chemotherapeutic agents frequently allows tumors to acquire a multidrug-resistant (MDR) phenotype $(3,4)$. Overexpression of MDR1, other ATP-dependent transporters, amplification of drug-inactivating enzymes, mutations or modifications of drug targets, alterations in DNA repair machinery and increased resistance to apoptosis cause this resistance (3-5). Toxicities of chemotherapy, along with drug resistance, are major therapeutic limitations that result in poor clinical outcomes in cancer patients.

Targeting of low extracellular $\mathrm{pH}$, elevated enzymes in tumor tissues, the hypoxic environment inside the tumor and tumor-specific antigens expressed on tumor cell surfaces were previously investigated as possible strategies for improved therapeutic outcomes (6-9). Singh et al highlighted recent trends in pro-drug and conjugate rationale and a design for cancer treatment, by analyzing comparative accounts of the advantages and disadvantages associated with each approach (10). A variety of receptors related to cellular growth factors or cytokines on tumor cells have been shown to be overexpressed (11), and we believe that targeting these receptors is a promising strategy. For example, the transfection of the tumor necrosis factor (TNF) receptor gene in cancer cells, or the exposure of cancer cells to certain reagents, may increase the expression of TNF receptors, resulting in the enhancement of the cytotoxic effect of TNF (12). Identifying new receptors on tumor cells, in addition to further investigation of therapeutic strategies, is still crucial for cancer treatment.

In the process of finding potential candidate receptors in cancer cells, the orexin 2 receptor (OX2R, also known as hypocretin receptor 2) was found to be a noteworthy target. The orexin family comprises orexins-A and -B, and their corresponding receptors are OX1R and OX2R, respectively. These 
two receptors belong to the seven-transmembraned G-coupled receptor superfamily (13). It has become clear that orexin receptors regulate narcolepsy $(14,15)$. Immunohistochemistry (IHC) analyses have demonstrated that certain peptides that bind to orexin receptors were selectively expressed in the hypothalamus, particularly in the lateral and medial hypothalamic regions (16).

At present, the presence of orexin receptors reported in human cancer cells is limited. The expression of OX1R has been found in cell lines from human colon cancer (17). However, the study pertaining to the expression of OX2R is limited to clinical samples of cortisol-secreting adrenocortical adenomas (18). Moreover, the role of orexin receptors in cancer cells is as yet unknown. Thus, identifying the location of orexin receptors remains a challenge.

OX2R was selected as a possible candidate since the expression levels of OX2R in normal cells are limited (16) and, thus, OX2R may be a cancer cell-specific target. Although a high expression of OX2R has been identified in hypothalamic samples, systemic administration of antitumor drugs targeting OX2R may not interact with the hypothalamus due to the presence of the blood-brain-barrier. In addition, orexin receptors are a well known target in the field of neuroscience, since they closely correlate with narcolepsy and other diseases $(14,15)$. Investigating other functions of OX2R would be helpful in understanding the roles of orexins.

In the present study, following the screening of OX2R expression in a variety of cancer cell lines, we investigated cancer tissue array samples to further examine OX2R expression. In addition, we examined the possibility of OX2R as a target for immunotoxin or antibody-drug conjugate (ADC) cancer therapy.

\section{Materials and methods}

Materials. Hepatocellular carcinoma tissue array, ARYHH0075 was purchased from Folio Biosciences (Columbus, OH, USA). Digestive system disease tissue array, DID381 was purchased from US Biomax (Rockville, MD, USA). Human cancer tissue array, VA2 was purchased from SuperBioChips (Seoul, Korea). All other reagents were of reagent grade quality. All tissue samples were collected under the ethical standards with the donor's complete informed consent under the control of manufacturers.

Polyclonal antibody against OX2R. Two New Zealand white rabbits were immunized with three specific peptides of OX2R to obtain a polyclonal antibody for OX2R. Three peptides (CRNWSSASELNETQE, FAHTEDRETVYAWF and C-AVAAEIKQIRARRK) were selected; GenBank reference: CAI19665.1. The immunization period was 64 days and immunization was performed four times during this period. Rabbit serum $(10 \mathrm{ml})$ were purified with a peptide affinity column. The antibody titer of ELISA for CRNWSSASELNETQE was estimated to be 1 in 4,000 and for C-AVAAEIKQIRARRK to be 1 in 16,000. All procedures were performed by Japan Bio Services (Saitama, Japan).

Reverse transcription polymerase chain reaction (RT-PCR). Total RNA was extracted according to the manufacturer's instructions (TRIzol, Invitrogen, Carlsbad, CA, USA). Following quantification of the extracted RNA, first strand complementary DNA (cDNA) of each cancer cell line was synthesized.

OX2R expression was measured by RT-PCR, using $1 \mu \mathrm{g}$ RNA and oligo (dT) as reverse transcription primers. A control reaction, which omitted reverse transcriptase was included to check for the presence of genomic DNA. OX2R was amplified using a Thermal Cycler (Applied Biosystems, Foster City, CA, USA) in a $20-\mu \mathrm{g}$ reaction medium containing $0.1 \mu \mathrm{g}$ of AmpliTaq Gold $^{\circledR}$ DNA Polymerase (Applied Biosystems), using the following cycling conditions: $95^{\circ} \mathrm{C}$ for $10 \mathrm{~min}$, followed by 35 cycles of $95^{\circ} \mathrm{C}$ for $30 \mathrm{sec}, 57^{\circ} \mathrm{C}$ for $30 \mathrm{sec}$ and $72^{\circ} \mathrm{C}$ for $60 \mathrm{sec}$, followed by a $10 \mathrm{~min}$ extension at $72^{\circ} \mathrm{C}$. The sequences for the sense and anti-sense primers of $\mathrm{OX} 2 \mathrm{R}$ and $\beta$-actin used were: 5'-caccgtgttcccaggcttag-3', 5'-ttctggctcggatctgcttt-3', 5'-cactgtgttggcgtacaggt-3' and 5'-tcatcaccattggcaatgag-3'. Amplicons were separated by electrophoresis in $2 \%$ agarose gel, stained with ethidium bromide and viewed under UV illumination. Gene sequence of OX2R was performed by Invitrogen Japan (Tokyo, Japan).

Western blotting. Proteins $(30 \mu \mathrm{g})$ were evaluated by the bicinchoninic acid (BCA) protein assay (Thermo Fisher Scientific, Waltham, MA, USA), loaded onto a NuPAGE 10\% Bis-Tris gel (Invitrogen) and blotted onto a Polyscreen polyvinylidene fluoride (PVDF) transfer membrane (ParkinElmer, Waltham, MA, USA) at $100 \mathrm{~V}$ for $1 \mathrm{~h}$ in a transfer buffer. The PVDF membranes were incubated with primary monoclonal antibody at a 1:2,000 dilution and with $\alpha$-tubulin antibody (ab24246, Abcam, Cambridge, MA, USA) at a 1:20,000 in phosphatebuffered saline (PBS)-0.1\% Tween (PBS-T) and $1 \%$ bovine serum albumin (BSA) overnight at $4^{\circ} \mathrm{C}$. The membranes were washed, incubated with a secondary anti-rabbit horseradish peroxidase-conjugated antibody (GE Healthcare, Fairfield, CT, USA) at a 1:20,000 dilution for $90 \mathrm{~min}$ at a room temperature and washed for $60 \mathrm{~min}$ with PBS-T. Antibody complexes were visualized using enhanced chemiluminenscense (ECL) plus western blotting reagent (GE Healthcare). The images were scanned by LAS-3000 UVmini (Fuji Film, Tokyo, Japan).

IHC. IHC was performed using tissue arrays. Tissue arrays were deparaffinized by xylene treatment and washed with an alcohol gradient (from 99 to 50\%) and PBS. Arrays were incubated with polyclonal antibody against OX2R at a 1:1,000 dilution overnight at $4^{\circ} \mathrm{C}$. Slides were developed with alkaline phosphatase (AP)-goat anti-rabbit IgG (Invitrogen) and stained by BM purple AP substrate (Roche Diagnostics, Rotkreuz, Switzerland). Slides were also lightly counterstained with hematoxylin and eosin. Negative controls in this study were prepared by omitting the primary antibody step.

Immunohistochemical scoring. Samples were scored independently by an experienced histopathologist. Immunostaining was scored as ++/+++ (strong), + (moderate) or - (negative).

\section{Results}

OX2R mRNA expression in cancer cells assessed by RT-PCR. One of the main objectives of this study was to confirm the expression levels of OX2R in cancer cells. We investigated 36 cancer cell lines originating from 10 different organs, ranging 
Table I. Cell lines used for RT-PCR and western blotting screening.

\begin{tabular}{ll}
\hline Organ & \multicolumn{1}{c}{ Cell line } \\
\hline SCCHN & YCUT231, YCUT891, YCUM 862, YCUM911, YCUMs861, YCUL891, Wmm-scc \\
Glioblastoma multiforme & SN19, U251tg, A172, U373, SF295 \\
Breast & BT-20, MDA-MB-231, ZR-75-1, T47D \\
Liver & Hep3B, HepG2, PLC/PFR-5, HuH-7 \\
Gallbladder & TGBC1, TGBC2, TGBC14, TGBC44, NOZ, OCUG \\
Cholangiocarcinoma & MzChA1,MzChA2, OZ, HuCCT-1 \\
Pancreas & PANC1, SU.86.86 \\
Uterine cervix & Hela, HT-29 \\
Oesophageal carcinoma & OE19 \\
Colon & DLD-1
\end{tabular}

RT-PCR, reverse transcription polymerase chain reaction; SCCHN, squamous cell carcinoma of the head and neck.

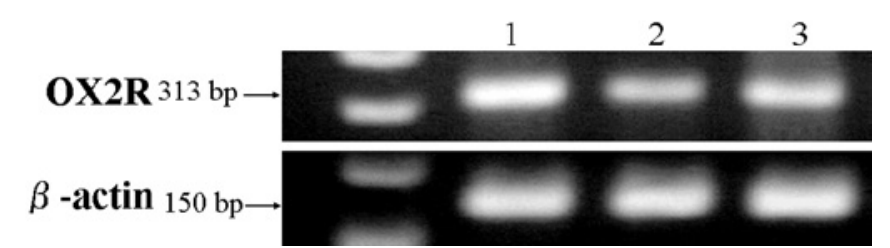

Figure 1. RT-PCR analysis of OX2R mRNA on cancer cells. Total RNA isolated from cancer cell lines (lane 1, TGBC2; lane 2, SF295 and lane 3, YCUM862) was reverse transcribed to cDNA and then assessed by PCR using specific primers as described in Materials and methods. $\beta$-actin was used as an internal control. Arrows show the location of amplified PCR products of OX2R and $\beta$-actin. OX2R, orexin 2 receptor; RT-PCR, reverse transcription polymerase chain reaction; cDNA, complementary DNA.

from glioblastoma to uterine cervix, and screened all 36 cell lines to assess this objective (Table I). Total RNA isolated from cancer cell lines was transcribed to cDNA, and then assessed by PCR as described in Materials and methods. Primer sequences and RT-PCR conditions were optimized by referring to other investigations analyzing the expression of OX2R $(19,20)$ and, through several trials, to obtain a clear single band.

Of the 36 cell lines, three cell lines, originating from the gallbladder (TGBC2), glioblastoma (SF295) and squamous cell carcinoma of the head and neck (YCUM862), expressed OX2R mRNA (Fig. 1). In addition, we performed gene sequences of PCR products to confirm its homology to OX2R. The homology between PCR products and OX2R was $98 \%$. We also screened for the expression of OX1R mRNA, but this was not identified in any of the cell lines tested in this study (data not shown).

$O X 2 R$ protein expression in cancer cells assessed by western blotting. To investigate the expression of $\mathrm{OX} 2 \mathrm{R}$ protein on cancer cells, we performed western blot analyses on the same cancer cell lines. Since antibodies against OX2R available for both western blotting and IHC were limited, we produced a polyclonal antibody against OX2R. We immunized two rabbits with three peptides specific to OX2R and purified serum as described in Materials and methods. Fig. 2 shows that all 3 cancer cell lines originated from the gallbladder (TGBC2), glioblastoma (SF295) and squamous

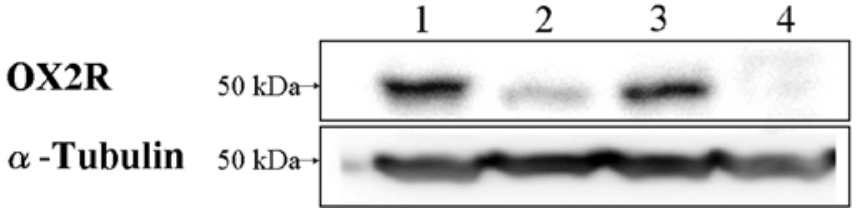

Figure 2. Expression levels of OX2R protein on cancer cells, which were found to be positive for OX2R mRNA upon RT-PCR analysis. Total cell lysates from cancer cells (lane 1, TGBC2; lane 2, SF295 and lane 3, YCUM862) were examined by western blot analysis as described in Materials and methods using specific antibody against OX2R. The extract from the OE19 cell line (lane 4), which was negative for OX2R mRNA expression in RT-PCR analysis was used as a negative control sample. $\alpha$-tubulin was used as the loading control. Arrows show the location of the molecular weight for OX2R and the $\alpha$-tubulin protein. OX2R, orexin 2 receptor; RT-PCR, reverse transcription polymerase chain reaction.

cell carcinoma of the head and neck (YCUM862), which were positive for OX2R mRNA expressed the protein of OXR2. Conversely, OX2R was not detected in the OE19 cell line, which did not express the mRNA of OX2R in RT-PCR analysis and was used as a negative control (Fig. 2). This result indicates that the three cell lines express OX2R.

OX2R expression in clinical tissue array samples. Based on the results of the mRNA and protein expression analysis, we performed an IHC analysis to analyze localization of OX2R on multiple organs and its frequency of expression among clinical cancer using the polyclonal antibody against OX2R shown at the western blot analysis stage. We prepared 221 clinical samples from the three tissue arrays purchased, which enabled us to obtain insights on the objectives described previously. Details of tissue arrays and protocols are described in Materials and methods.

As shown in Table II, 69 of the 221 samples expressed OX2R, including 8 samples with strong staining and 61 samples with moderate staining. The remaining 152 samples did not react with the OX2R antibody. We observed that the expression of OX2R was located on the cell membrane, where receptors should be located, as shown in Fig. 3. These results suggest that $31.2 \%$ of clinical cancer samples express OX2R on their 
Table II. Summary of OX2R immunohistochemistry analyses in a variety of cancers using three tissue arrays.

\begin{tabular}{|c|c|c|c|c|c|}
\hline \multirow[b]{2}{*}{ Organ } & \multirow[b]{2}{*}{ No. of patients } & \multicolumn{3}{|c|}{ Staining intensity } & \multirow[b]{2}{*}{ Positive samples (\%) } \\
\hline & & - & + & $++/+++$ & \\
\hline Liver & 80 & 71 & 7 & 2 & 11 \\
\hline Stomach & 34 & 16 & 17 & 1 & 53 \\
\hline Colon & 20 & 13 & 7 & 0 & 35 \\
\hline Salivary gland & 15 & 8 & 6 & 1 & 47 \\
\hline Larynx & 15 & 7 & 7 & 1 & 53 \\
\hline Rectum & 9 & 6 & 2 & 1 & 33 \\
\hline Lung & 9 & 8 & 1 & 0 & 11 \\
\hline Esophagus & 6 & 6 & 0 & 0 & 0 \\
\hline Small intestine & 6 & 0 & 5 & 1 & 100 \\
\hline Nasopharynx & 6 & 4 & 2 & 0 & 33 \\
\hline Pancreas & 6 & 3 & 3 & 0 & 50 \\
\hline Gallbladder & 5 & 2 & 3 & 0 & 60 \\
\hline Tongue & 2 & 2 & 0 & 0 & 0 \\
\hline Maxilla & 2 & 0 & 1 & 1 & 100 \\
\hline Bile duct & 2 & 2 & 0 & 0 & 0 \\
\hline Mandible & 1 & 0 & 1 & 0 & 100 \\
\hline Lower lip & 1 & 1 & 0 & 0 & 0 \\
\hline Tonsil & 1 & 1 & 0 & 0 & 0 \\
\hline Anus & 1 & 1 & 0 & 0 & 0 \\
\hline Total & 221 & 151 & 62 & 8 & \\
\hline
\end{tabular}

OX2R, orexin 2 receptor.

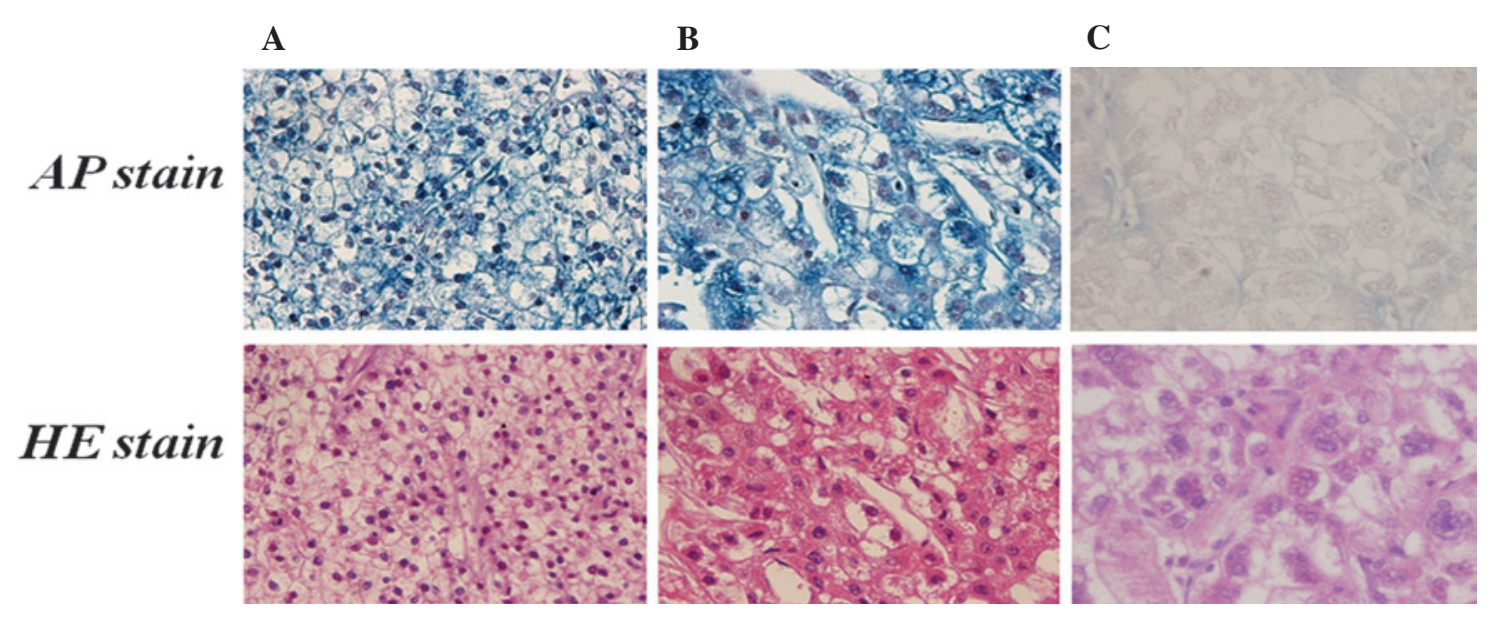

Figure 3. Immunohistochemistry analysis for OX2R expression in various types of cancer. Tissue specimens on arrays were stained with hematoxylin and eosin (H\&E) or with alkaline phosphatase (AP), which binds specific polyclonal antibodies to OX2R. (A) Images from sample Folio ARY-HH0075 C6 (hepatocellular carcinoma; original magnification, $\mathrm{x} 200$ ). (B) The magnified immunohistochemical image shows the staining for OX2R is significantly limited on the cell membrane. (Folio ARY-HH0075 G3 sample; original magnification, x400). (C) Images of Folio ARY-HH0075 C4 as a negative control (original magnification, $\mathrm{x} 400)$. OX2R, orexin 2 receptor.

cell membrane. It is also assumed that cancer organs such as the stomach, salivary gland and larynx expressed OX2R more frequently than other organs.

\section{Discussion}

This study showed the presence of OX2R on several clinical cancer samples including salivary gland, stomach and small intestine cancer. We demonstrated the existence of OX2R on cell lines as well as on clinical samples using tissue arrays. These results suggest that OX2R is overexpressed in certain types of cancer and may have biological roles in cancer cells. To reveal the functions of OX2R in cancer cells, we conducted additional experiments to confirm the reaction of OX2R-positive cells with a ligand, orexin B. We observed minimal growth promotion of OX2R-positive cancer cells despite increasing ligand 
doses (data not shown). Thus, it is suggested that orexin B does not affect the growth of OX2R-expressing cancer cells and that traditional molecular-targeted approaches, such as anti-OX2R antibodies, may not work effectively. Reacting specifically to OX2R and exhibiting toxicity only inside the OX2R-expressing cells may be criteria for drug candidates to target $\mathrm{OX} 2 \mathrm{R}$ as a potential mode of action.

Based on insights revealed in this study, we believe that antibodies or ligands conjugated to natural toxins or chemicals, termed immunotoxins or ADCs (21-23), are likely to be an ideal strategy to target receptors such as OX2R. Immunotoxin or ADCs is likely to be an appropriate approach to treat OX2Rpositive cancer cells, since antibodies or ligands that bind OX2R themselves are often non-cytotoxic, as previously noted. Immunotoxin is a rationally designed anticancer agent with potent toxins that target cell-surface antigens or receptors on cancer cells. An immunotoxin strategy enables the OX2R antibody to add specific cytotoxicity to cancer cells by fusing the $\mathrm{OX} 2 \mathrm{R}$ antibody to toxins. This strategy may also avoid toxicity to the hypothalamus where OX2R is normally expressed with the function of the blood-brain-barrier to maintain homeostasis $(24,25)$. In addition to the direct killing of cancer cells, antibody-dependent cell-mediated cytotoxicity (ADCC) occurs if the antibody to OX2R is conjugated with toxic moiety.

OX2R immunotoxin is an alternative option for patients with resistance to current antibody therapies, since its antitumor effect varies from current molecular-targeting drugs. Moreover, dual-specific immunotoxin enables the enhancement of this type of multi-anti-tumor effect. Conjugating the OX2R antibody and kinase inhibitors may elicit both the ADCC and multi-kinase inhibition effects.

In this study, the presence of OX2R on a variety of types of cancer was suggested. However, further studies are required to confirm the presence of $\mathrm{OX} 2 \mathrm{R}$, since the number of clinical samples in the same organs are limited. At present, additional IHC experiments are underway using a larger number of clinical samples to confirm statistical significance of the presence of $\mathrm{OX} 2 \mathrm{R}$ and the correlation with stage and cancer type. Therefore, further investigations on OX2R and new immunotoxin/ADC approaches may offer new therapeutic options for cancer patients.

\section{Acknowledgements}

We thank Nana Kawaguchi and Kumi Kodama (Department of Pharmacoepidemiology, Kyoto University) for technical assistance with cell culturing and Motomichi Matsuzaki (Department of Biomedical Chemistry, The University of Tokyo) for advice on immunohistochemistry.

\section{References}

1. Hickman JA: Apoptosis induced by anticancer drugs. Cancer Metastasis Rev 11: 121-139, 1992.

2. Andersson A, Fagerberg J, Lewensohn R and Ehrsson H: Pharmacokinetics of cisplatin and its monohydrated complex in humans. J Pharm Sci 85: 824-827, 1996.

3. Szakacs G, Paterson JK, Ludwig JA, Booth-Genthe C and Gottesman MM: Targeting multidrug resistance in cancer. Nat Rev Drug Discov 5: 219-234, 2006.
4. Wilson TR, Longley DB and Johnston PG: Chemoresistance in solid tumours. Ann Oncol 17: 315-324, 2006.

5. Sharom FJ: ABC multidrug transporters: structure, function and role in chemoresistance. Pharmacogenomics 9: 105-127, 2008.

6. Izumi $\mathrm{H}$, Torigoe $\mathrm{T}$, Ishiguchi $\mathrm{H}$, Uramoto $\mathrm{H}$, Yoshida $\mathrm{Y}$, Tanabe $\mathrm{M}$, et al: Cellular $\mathrm{pH}$ regulators: potentially promising molecular targets for cancer chemotherapy. Cancer Treat Rev 29: 541-549, 2003.

7. Husain I, Mohler JL, Seigler HF and Besterman JM: Elevation of topoisomerase I messenger RNA, protein, and catalytic activity in human tumors: demonstration of tumor-type specificity and implications for cancer chemotherapy. Cancer Res 54: 539-546, 1994.

8. Liu H, Savaraj N, Priebe W and Lampidis TJ: Hypoxia increases tumor cell sensitivity to glycolytic inhibitors: a strategy for solid tumor therapy (Model C). Biochem Pharmacol 64: 1745-1751, 2002.

9. Boon T and Old LJ: Cancer Tumor antigens. Current Opinion in Immunology 9: 681-683, 1997.

10. Singh Y, Palombo M and Sinko PJ: Recent trends in targeted anticancer prodrug and conjugate design. Curr Med Chem 15: 1802-1826, 2008

11. Koji K, Oumi N, Ryuichi M and Ryozo N: Targeted anticancer immunotoxins and cytotoxic agents with direct killing moieites. Sci World J 6: 781-790, 2006.

12. Ohara H, Hasegawa $Y$, Kawabe $T$, Ichiyama $S$, Hara $T$, Shimono Y, et al: Effect of gene transfer of tumor necrosis factor receptors into human lung carcinoma cell line. Jpn J Cancer Res 89: 589-595, 1998.

13. Sakurai T, Amemiya A, Ishii M, Matsuzaki I, Chemelli RM, Tanaka H, et al: Orexins and orexin receptors: a family of hypothalamic neuropeptides and $\mathrm{G}$ protein-coupled receptors that regulate feeding behavior. Cell 92: 573-585, 1998.

14. Peyron C, Faraco J, Rogers W, Ripley B, Overeem S, Charnay Y, et al: A mutation in a case of early onset narcolepsy and a generalized absence of hypocretin peptides in human narcoleptic brains. Nat Med 9: 991-997, 2000.

15. Thannickal TC, Moore R, Nienhuis R, Ramanathan L, Gulyani S, Aldrich $\mathrm{M}$, et al: Reduced number of hypocretin neurons in human narcolepsy. Neuron 27: 469-474, 2000.

16. Nambu T, Sakurai T, Mizukami K, Hosoya Y, Yanagisawa M and Goto K: Distribution of orexin neurons in the adult rat brain. Brain Res 827: 243-260, 1999.

17. Rouet-Benzineb P, Rouyer-Fessard C, Avondo V, Pouzet C, Yanagisawa $\mathrm{M}$, et al: Orexin acting at native $\mathrm{OX} 1$ receptor in colon cancer and neuroblastoma cells or at recombinant OX1 receptor suppress cell growth by inducing apoptosis. J Biological Chem 279: 45875-45886, 2004.

18. Spinazzi R, Rucinski M, Neri G, Malendowicz LK and Nussdorfer GG: Preproorexin and orexin receptors are expressed in cortisol-secreting adrenocortical adenomas, and orexins stimulate in vitro cortisol secretion and growth of tumor cells. J Clin Endocrinol Metab 90: 3544-3549, 2005.

19. Karteris E, Chen $\mathbf{J}$ and Randeva $\mathrm{H}$ : Expression of human prepro-orexin and signaling characteristics of orexin receptors in the male reproductive system. J Clin Endocrinol Metab 89: 1957-1962, 2004.

20. Digby JE, Chen J, Tang JY, Lehnert H, Matthews RN and Randeva HS: Orexin receptor expression in human adipose tissue: effects of orexin-A and orexin-B. J Endocrinology 191: 129-136, 2006.

21. Reiter Y: Recombinant immunotoxins in targeted cancer cell therapy. Adv Cancer Res 81: 93-124, 2001.

22. Pastan I, Chaudhary V and FitzGerald DJ: Recombinant toxins as novel therapeutic agents. Annu Rev Biochem 61: 331-354, 1992.

23. Johnson DA and Laguzza BC: Antitumor xenograft activity with a conjugate of a Vinca derivative and the squamous carcinoma-reactive monoclonal antibody PF1/D. Cancer Res 47: 3118-3122, 1987.

24. Levin VA: Relationship of octanol/water partition coefficient and molecular weight to rat brain capillary permeability. J Med Chem 23: 682-684, 1992

25. Sun H, Dai H, Shaik N and Elmquist WE: Drug efflux transporters in the CNS. Adv Drug Delivery Rev 55: 83-105, 2003. 